\title{
La mujer ecuatoriana en las Fuerzas Armadas: un espejo de la sociedad
}

\author{
Editado por/Edited by: Johanna Andrango \\ Recibido/Received: 9/11/2014. Aceptado/Accepted: 16/05/2015 \\ Publicado en línea/Published on Web: 16/10/2015
}

\author{
María del Carmen Iturralde \\ Universidad San Francisco de Quito, Colegio de Ciencias Sociales y Humanidades COCISOH \\ Diego de Robles y Vía Interoceánica, Quito, Ecuador
}

Correo Electrónico:miturralde_v@hotmail.com

\section{Resumen}

En la actualidad la mujer representa un elemento humano fundamental en el Ejército ecuatoriano. Paulatinamente, la participación de las mujeres en las Fuerzas Armadas se presenta cada vez más profesional en similares condiciones de sus compañeros varones. En la medida que la mujer se involucra en el ámbito militar procura guardar una correlación cercana con la forma en la que se desenvuelve en el mundo civil. Es decir, el rol que la mujer cumpla dentro de la sociedad ecuatoriana será el rol que cumpla dentro de las instituciones militares. Según el 'feminismo posmoderno', es evidente que los roles tanto de hombres como mujeres son ambiguos, debido a que son creados y reforzados todos los días por cada uno de los miembros de una sociedad. Por lo tanto es posible decir que toda institución es el reflejo de su sociedad, y en ese caso las FFAA no son la excepción. Las diferencias entre hombres y mujeres definitivamente existen; sin embargo, debe entenderse que estas diferencias han sido socialmente construidas desde hace siglos atrás. Las FFAA, a su vez, usan estas diferencias para contrarrestar los efectos de la guerra a nivel mundial utilizando las cualidades que se han caracterizado como femeninas.

Palabras clave: Feminismo, mujer ecuatoriana, Ecuador, Fuerzas Armadas, constructivismo, ejército, género, construcción social.

\section{Abstract}

Nowadays, women represent a key element in the Ecuadorian Army. Gradually, the role of women in the Armed Forces becomes more professional compared to their male coworkers. The way women gets involved in the military world, has a direct correlation to the way that they behave in the civilian world and the role that woman has within the Ecuadorian society meets the role within the Military Institutions. According to 'postmodern feminism', it is clear and evident that the roles of both men and women are ambiguous, given that they are created and reinforced every day by every member of a society. Therefore, it is possible to say that every institution is a reflection of the society it exists in, and the case of the Ecuadorian armed forces is not the exception. The differences between men and women do exist and are definitely present; nevertheless, it must be understood that these differences were socially constructed centuries ago. The Ecuadorian armed forces use these differences to counteract the effects of war at a global level, using qualities that have been socially characterized and accepted as female qualities.

Keywords: Feminism, Ecuadorian women, Ecuador, Armed Forces, constructivism, Army, gender, social construction.

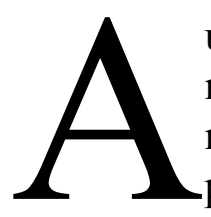

ún resulta extraño para muchos ver a una mujer ecuatoriana uniformada en su camuflaje, con botas, y llevando distintivos pegados a su uniforme. Sin embargo, puede resultar más extraño - aún para algunos ecuatorianos - el saber que hoy en día algunas de ellas podrían participar activamente dentro del campo de batalla, y no solamente del proceso logístico y administra- tivo de las Fuerzas Armadas Ecuatorianas (Chacón, 2008). Pese a la construcción del significado de género a lo largo de la historia, en los imaginarios sociales la mujer ha continuado siendo proyectada como un individuo social débil de género y vulnerable. Esta imagen femenina ha sido considerada como un sujeto que requiere de protección, y poseedor de una postura victimizada. Siempre ha sido ignorado el hecho 
de que a lo largo de la historia de nuestro país las mujeres han sido parte inherente e innegable de los conflictos armados que lo marcaron.

Las guerras en el Ecuador -así como en el resto del mundo- parten de la idea casi intrínseca de que son ejecutadas y resueltas exclusivamente por soldados varones, mientras que el papel de las mujeres se reduce a esperar a sus maridos en casa, cuidar de los niños y ser víctimas distantes del conflicto. Sin embargo, las mujeres han construido la historia de nuestro país en lo que se refiere a conflictos armados, inclusive desde antes de la existencia del Ecuador como República.

Es así que, el papel de la mujer en cuestiones políticas y militares se registró desde la época colonial con María Caiche, quien llegó a ser cacique de Daule y Quijos e hizo que los dauleños dejen de trabajar sin paga para los líderes españoles. Por otra parte, en las guerras de independencia es casi imposible olvidar a las "Tres Manuelas" quienes dejaron del lado el rol femenino impuesto y se dedicaron a liberar a su pueblo de imposiciones extranjeras para comenzar a construir un país libre (Chacón, 2008).

El tema de la inclusión de las mujeres dentro de las Fuerzas Armadas del Ecuador ha surgido recientemente, debido a que no hace mucho tiempo atrás las mujeres fueron aceptadas por primera vez en la Escuela Superior Militar Eloy Alfaro (ESMIL). Actualmente, en las FFAA las mujeres reciben el mismo trato y tienen los mismos derechos que los hombres, pero el cambio en esta institución -tradicionalmente masculina- se ha presentado gracias a los cambios sucedidos dentro de la estructura general de la sociedad ecuatoriana.

Las instituciones modifican la forma en la que la sociedad se construye, y de la misma manera, la construcción de la sociedad dicta la dirección en la que se modifican las instituciones, por lo tanto, si es que no es bien visto que una mujer trabaje, habrá más mujeres en casa que trabajando, y si a la mujer se le prohíbe estudiar, entonces el poder que brinda el conocimiento va a estar concentrado en manos masculinas. De igual manera, si la mujer va más allá de los roles tradicionales de género, es entonces que las instituciones se ven obligadas a cambiar y a acoger las nuevas normas de la sociedad. Esto afirmo no como un juego de palabras, sino como una realidad.
A partir de esta breve introducción se da inicio a la tesis central del presente artículo, la cual sostiene que la cultura dicta de qué forma se mueve el mundo en el que vivimos, y con las instituciones políticas o gubernamentales no es diferente, por lo que podemos decir que las Fuerzas Armadas como institución son el reflejo de la sociedad, la cultura y las reglas.

El rol de género en conflictos armados ha sido usualmente visto desde tres ramas feministas fundamentales: el feminismo liberal, que radica en que las mujeres y los hombres son totalmente iguales, y no hay diferencias ni biológicas ni psicológicas que los separen, y el feminismo de la diferencia, que argumenta que los hombres y las mujeres son completamente diferentes, pero esas diferencias tienen que ser celebradas y no usadas para la marginalización de uno de los dos géneros. Una tercera teoría del feminismo, y la que será usada en este análisis es el feminismo posmoderno, donde las diferencias de género radican en construcciones culturales arbitrarias (Goldstein, 2001).

Esta idea constructivista defiende el hecho de que el rol de género es una construcción social y cultural, que los hombres y las mujeres inconscientemente aprendemos a actuar de cierta forma por influencia de las normas sociales, y además las instituciones que nos rodean obedecen al comportamiento colectivo de las personas que conformamos una cierta sociedad. Como un gran ejemplo se puede mencionar la incógnita en Ciencia Política que radica en cómo organizar a una sociedad para que sus funciones sean democráticas; por una parte, se puede decir que se necesitan instituciones fuertes que regulen los mecanismos democráticos existentes, y por otra parte se puede decir que se necesita una modificación en la cultura política. Si analizamos la existencia de las mujeres en las Fuerzas Armadas, podemos decir que el funcionamiento de las instituciones responde al comportamiento de sus actores; por lo tanto las Fuerzas Armadas del Ecuador son el reflejo de la sociedad ecuatoriana, en donde la institución se modifica a la par de la sociedad.

Basado en la idea de Sartre de que la naturaleza humana no es única ni es la misma en todos lados (Hopkins, 1968), y en el constructivismo social en donde la sociedad y las instituciones se construyen mutuamente, se puede decir que la mujer ecuatoriana 
cumple con el rol que la sociedad le ha impuesto. En las FFAA la mujer se ha insertado en la institución de la misma forma que ha ocupado cualquier otro puesto de trabajo o roles políticos, profesionales, educativos y económicos; además cumple su función de soldado y al mismo tiempo de mujer según lo que dicta la sociedad. Este artículo pretende hacer una revisión de las Fuerzas Armadas del Ecuador como una institución que se ve modificada a la par de la sociedad, mediante un análisis histórico, sociológico y constructivista de la participación de la mujer en la milicia desde el siglo XIX.

\section{Rol femenino de Género}

En el siglo XIX las mujeres participaron activamente de las luchas alfaristas, sobre todo a favor de la revolución liberal y de los ideales que les otorgarían derechos semejantes a los de los hombres en una sociedad profundamente machista. Los grupos organizados de gente con ideales revolucionarios en la época de Alfaro fueron llamados "montoneros" y, a pesar de que en este tiempo la idea de una mujer en protesta era casi inconcebible, algunas "guarichas" dejaron atrás los prejuicios de la época y se sumaron a la revolución liberal. (De acuerdo con la Real Academia Española, una guaricha era la mujer valiente y decidida que acompañaba a su hombre cuando emprendía la marcha a hacia el combate y ella camina detrás de los repartos armados.)

Ya en la segunda mitad del siglo XX, desde el año 1956, ingresaron esporádicamente algunas mujeres a ser parte de las Fuerzas Armadas ecuatorianas. Sin embargo, su entrada a la institución no era regular debido a que no eran graduadas en las escuelas militares, solo ingresaban a cumplir con ciertas misiones especialistas ya que no existía un marco legal para que ingresen de manera regular (Chacón, 2008).

La idea de una mujer revolucionaria en la época del Eloy Alfaro no era bien vista, ya que el conservadurismo englobaba absolutamente toda práctica social, desde la religión hasta la política. De igual manera, los roles tradicionales se han implantado tanto en la sociedad ecuatoriana, que para que un hombre sea considerado hombre, ha tenido que mantener su imagen de cabeza de familia, de quien todos dependen, entre otros estereotipos. Estos roles, sin duda en menor grado, se mantienen hasta el día de hoy.
Se puede llegar a deducir que - según el pensamiento de esa época- si el hombre es aquel que construye la sociedad de una manera productiva, entonces también es él quien tiene que encargarse de la guerra. Estos roles tradicionales dividen a los hombres y a las mujeres de su calidad de humanos, sin embargo resulta difícil distinguir si es que las diferencias entre hombres y mujeres son exógenas (resultado de lo exterior), o endógenas (resultado de sí mismo) (Ichino $\&$ Moretti, 2009). Desde un punto de vista sociológico, los roles de género son exógenos, construidos por la sociedad, hechos por los seres humanos, por lo tanto una mujer debería tener las mismas cabidas en la guerra que un hombre, pero debido a su rol histórico, estas se ven distorsionadas y disminuidas, tanto por los hombres como por las mismas mujeres.

En el año 1999 ingresaron a la Escuela Superior Militar 'Eloy Alfaro' las primeras cadetes mujeres para convertirse en subtenientes de Servicios; aunque eran once, alrededor de la mitad se graduó después de cuatro años de formación militar, tal y como lo haría un cadete varón. La ESMIL recibe anualmente alrededor de mil aspirantes a cadetes, de las cuales entran sólo 250 después de realizar evaluaciones psicológicas, académicas, médicas y físicas. No hay diferencias entre los cupos de hombres y de mujeres pero anualmente no ingresan más de 20 aspirantes mujeres ya que no hay gran demanda y no todas cumplen con los requerimientos necesarios para el ingreso a un régimen militar.

Las primeras promociones de mujeres que se graduaron de la ESMIL han realizado el curso denominado 'Tigres' a la par de sus compañeros hombres, el cual requiere un gran esfuerzo físico y es otro requisito para ascender a la jerarquía de teniente. Así mismo, algunas de las primeras mujeres graduadas de la ESMIL alcanzaron las primeras antigüedades en el 'Curso Básico' -requisito para ascender a capitán- y están a punto de ingresar al Curso Avanzado para ser promovidas al grado de mayor. Según estadísticas de la Red de Seguridad y Defensa de América Latina del año 2012, hay 193 mujeres que sirven en el Ejército Ecuatoriano, y contando los integrantes de las tres ramas de FFAA, alrededor del 1,6\% son personal femenino (647 personas) (Donadio, M., 2012).

Cabe recalcar que las mujeres siempre han estado involucradas de una manera un tanto informal 
en los conflictos armados, ya que supieron en algunos casos aportar a la Inteligencia Militar siendo una herramienta de obtención de información útil para la institución castrense. Actualmente esos papeles un tanto improvisados se han vuelto parte del rol que tienen las mujeres ecuatorianas en las Fuerzas Armadas. En Ecuador, las mujeres no son de armas de combate, son más bien de armas de apoyo, lo que quiere decir que ayudan en la guerra sin estar físicamente en el campo de batalla y en el momento de los ataques; sin embargo, la Fuerza Aérea Ecuatoriana si cuenta con mujeres con ciertos elementos de combate y en ciertas circunstancias, como por ejemplo que hay pilotos mujeres preparadas para pilotear aeronaves de combate o de apoyo al combate.

Dentro de la Fuerza Terrestre las mujeres pueden especializarse en diferentes ramas; por ejemplo, pertenecer a las operaciones de Inteligencia Militar y servir a la fuerza haciendo indagaciones y protegiendo la información del país. Las mujeres también pueden pertenecer al ámbito de Comunicaciones, esto significa que planean y facilitan la comunicación entre los mandos y las tropas, tan importante en el desempeño bélico. Las mujeres dentro de la Fuerza Terrestre también pueden aspirar a ser de servicios, lo que significa que abastecen de alimento, transporte, equipamiento, munición y armamento a los soldados en guerra, se encargan del mantenimiento y la logística de instalaciones y en la franca y ordenada movilización de tanques y vehículos. También -y con mucho éxito- pueden ser del Servicio de Sanidad, o sea médicos que sirven a los soldados en distintos accidentes que suceden en una guerra (Chacón, 2008).

Las mujeres de la Fuerza Terrestre pueden aspirar a ser parte del arma de Ingeniería, lo que significa que preparan el terreno para la guerra y proporcionan movilidad para los soldados construyendo y destruyendo distintos obstáculos. Por otra parte se encargan también de la contra movilidad, esto significa que colocan obstáculos para dificultar el paso del enemigo. Dentro del área de Comunicación Social, las mujeres manejan operaciones psicológicas, en donde se utilizan distintas estrategias que aportan a la guerra ya que, dentro de un conflicto armado, pues hay batallas que se ganan únicamente con estrategia sin el uso de la fuerza. Actualmente en la FAE las mujeres cadetes pilotos se preparan para volar helicópteros y aviones subsónicos y en pocos años, cuando logran cumplir todos los prerrequisitos, pueden volar aviones supersónicos activamente en caso de guerra.

\section{Roles Tradicionales y Estereotipos}

La mujer a nivel mundial ha tomado tradicionalmente un rol pasivo, de fragilidad y debilidad, de desprotección, de un ser vulnerable, pacífico y cuidadoso. El hombre, por su parte, siempre ha sido considerado como el ejecutor de toda acción importante, fuerte, protector, agresivo, valiente, el hombre siempre ha sido el héroe (Caprioli \& Boyer, 2001). Estos roles se han visto reflejados tanto en la historia de las FFAA, como en las instituciones deportivas, religiosas, políticas, familiares, educativas y económicas. Por ende, a medida de que estos roles se desvanecen más y más, estas instituciones cambian de igual forma como una consecuencia de los avances de la sociedad.

Los conflictos armados, en donde hombres y mujeres han participado activamente a lo largo de la historia, siempre han tenido objetivos y fines políticos, donde los hombres han sido tradicionalmente quienes tienen dominancia económica, social y política sobre la mujer. Los estereotipos de la cultura latinoamericana radican en que la mujer es débil y no puede participar de un conflicto armado porque, además de ser inútil, no corresponde a ese lugar, mientras que los varones pertenecen a todo aquello donde hay violencia. Estos prejuicios son completamente injustos, tanto para la imagen de las mujeres limitadas a seguir con lo que impone la sociedad, como para los varones, quienes son sutilmente obligados a la violencia para ser "hombres".

En el año 2007 Guadalupe Larriva -primera mujer y civil en ser Ministro de Defensa del Ecuador-, anunció en su posición en esa cartera de Estado, un proyecto de fronteras vivas en los límites con Colombia y Perú, además criticó por las intervenciones militares en el Plan Colombia y de la Base Militar Estadounidenses en Manta (El Comercio, 2014). A pesar de que un trágico accidente terminó con su vida al poco tiempo de posicionarse, cabe recalcar que Larriva ocupó un puesto que solo estuvo a cargo de hombres, y tradicionalmente lo ocupaban generales y militares retirados. La primera ministra rompió completamente con los estereotipos impuestos y con una tradición inminente de las FFAA, inclusive tras su fallecimiento otra mujer ocupó esa función. 
Las FFAA cambian a la par de la sociedad, entonces si la sociedad "permite" que las mujeres hagan lo mismo que hacen los hombres, las FFAA también incentivan a la igualdad de género, así como en otras instituciones.

\section{Diferencias entre hombres y mujeres militares}

Se suele pensar $-\mathrm{y}$ hasta se dice- que solo los hombres están a cargo de la guerra, y que los efectos de ésta serían mucho peores que si una mujer es parte del conflicto, ya que las mujeres están orientadas hacia la paz (Mark Tessler, 1999). El 'feminismo de la diferencia' afirma que los hombres y las mujeres son inherentemente diferentes ya que mientras los hombres se encuentran aptos para la guerra, la naturaleza de las mujeres provoca que resuelvan los conflictos sin llegar a la guerra. Esta rama del feminismo argumenta que las mujeres tienen más experiencia en la resolución diplomática de conflictos y en las relaciones interpersonales que los hombres, mientras que los hombres son más aptos para participar en un combate que las mujeres (Mark Tessler, 1999). Si volvemos al pensamiento de Sartre en donde se obvia la existencia de la naturaleza humana las diferencias entre hombres y mujeres aún están presentes, y esto se debe a una construcción social en donde el género femenino se caracteriza por ser más sensible, cuidadoso y maternal que el masculino, ya que eso es lo que han aprendido desde su nacimiento con la influencia de la sociedad.

Al estar claras estas diferencias, se puede explicar el hecho de que una mujer en las FFAA no solo puede preparar los diferentes componentes de guerra en el país sino que puede humanizar el conflicto y además puede evitar el llegar a una guerra en términos de diplomacia. Se propone que sin la ayuda de la mujer en conflictos armados en el Ecuador, los problemas bélicos podrían ser mucho más violentos. Esta explicación también responde a la inserción de mujeres en las Operaciones de Mantenimiento de Paz de la ONU, ya que la intervención de una mujer puede ser mucho más efectiva que la de un hombre.

El Ecuador es un país que envía peacekeepers de Misiones de Paz en países, como Haití, Liberia, Sudán y Costa de Marfil, entre otros. La violencia sexual en contra de las mujeres es un arma que ha sido comúnmente usada alrededor del mundo desde hace muchos siglos para debilitar al opositor. Según
Nicola Duckworth, directora del Programa para Europa y Asia Central de Amnistía Internacional, durante el conflicto de Bosnia y Herzegovina de 1992 a 1995, "miles de mujeres y niñas fueron violadas, a menudo con una brutalidad extrema; muchas fueron recluidas en campos penitenciarios, hoteles y domicilios particulares donde fueron sometidas a explotación sexual. Muchas mujeres y niñas fueron asesinadas" (Amnesty International, 2009).

Actualmente, muchos países en grandes situaciones de guerra tienen este problema; las mujeres son violadas y están desprotegidas de la sociedad y el sistema. Para ayudar a solucionar problemas de este tipo, es necesaria la ayuda de oficiales mujeres especialistas en el mantenimiento de paz. El Ecuador cuenta solamente con una mujer peacekeeper que estuvo de misión de Haití, pero se necesitan muchas más para ayudar al desarrollo de las mujeres que viven en países con conflictos críticos y bajo sus estragos bélicos (Donadio, M., 2012).

\section{EI espejo de la sociedad}

Si los cambios que favorecían a las mujeres ecuatorianas dentro de la institución armada en el Ecuador llegaron a realizarse, es nada más y nada menos porque toda la sociedad ecuatoriana cambió con respecto a su posición en los roles de género. Dentro de las FFAA ecuatorianas no hay nada que impida que las mujeres lleguen -corridos los tiempos y las exigencias comunes- al máximo grado de General de la Republica, al igual que los hombres, por lo tanto todos se enfrentan a la misma competencia. La gran diferencia actualmente entre hombres y mujeres en el mundo militar es el número, ya que hay un $1,6 \%$ de mujeres frente a un $98,4 \%$, pero así mismo hay menos mujeres que hombres que son dueñas de sus empresa, que están involucradas en altos puestos de trabajo, en la política, o que poseen inmuebles. No hay ningún factor que impida el progreso de la mujer en las fuerzas armadas; el hecho de que hayan más hombres que mujeres en la institución armada es más bien es un factor cultural. Sin embargo, a medida de que el rol de la mujer se transforme en la sociedad, también las FFAA cambiarán al mismo ritmo.

Del mismo modo, si es que la sociedad se caracteriza por ser patriarcal y machista, estas características también estarán reflejadas en la institución militar; no en las políticas institucionales, pero sí en 
las actitudes algunos oficiales hombres. En una entrevista realizada en abril de 2014 a 6 cadetes de la ESMIL a cargo de la teniente Liliana Salazar, ellas hablaron acerca del machismo en la institución y la conclusión fue que no se puede esperar que una institución tradicionalmente de hombres sea perfecta desde el principio si es que la sociedad no ha cambiado su mentalidad en lo que respecta a igualdad de género. Como ellas mismo expresaron, lo que le hace falta al país es una demostración de que la mujer es exactamente igual al hombre y con el tiempo esta percepción cambiará. Una institución ecuatoriana, conformada por ecuatorianos y que sirve al Ecuador es completamente influenciada por la sociedad a la que hace parte.

En la ESMIL se trata de aislar las expresiones de machismo debido a la estructura normativa y jerárquica que existe. Si una cadete u oficial mujer lleva más tiempo (o tiene más experiencia) en la carrera militar (tiene mejores calificaciones o tiene un rango superior) que sus compañeros, ya sean varones o mujeres, ellos le deben respeto por ley dentro de la institución militar. A pesar de ello, las cadetes entrevistadas mencionaron que algunos de sus compañeros creen que los hombres son superiores, y se molestan, en especial, cuando las mujeres obtienen calificaciones más altas en el ámbito académico, teniendo en cuenta la meritocracia que existe dentro de la milicia.

\section{Conclusión}

La mujer ecuatoriana, a la par de sus compañeros hombres, ha sido y será siempre parte fundamental del ejército ecuatoriano, cada vez más profesional que antes. La medida en la que una mujer se involucra en el ámbito militar depende de la forma en la que se involucra en el mundo civil; el rol que la mujer cumpla dentro de la sociedad ecuatoriana es el rol que cumplirá en las instituciones militares. Se debe señalar que esta institución está conformada por hombres y mujeres ecuatorianos, criados bajo la misma sociedad y educados de manera similar, por lo que la institución será el reflejo de la sociedad colectiva. Los cambios que se dan en el Ecuador como colectivo afectan a todas las instituciones ecuatorianas, entre ellas, las FFAA.

Según el feminismo posmoderno, es evidente que los roles son ambiguos debido a que son creados y construidos por la misma comunidad. Además, des- cartando la existencia de una naturaleza humana, es posible decir que toda institución es el reflejo de su sociedad, por lo que funciona de la misma manera y se presentan los mismos roles, los mismos estereotipos, y las mismas diferencias; en este caso, las FFAA no son la excepción. No se puede negar que existan diferencias entre hombres y mujeres; definitivamente existen y son más que evidentes, no obstante se debe entender que estas diferencias han sido construidas socialmente desde hace siglos atrás. Las FFAA, usan estas diferencias para contrarrestar los efectos de la guerra internamente y a nivel mundial por medio de agentes de paz desplegadas en misiones alrededor del mundo.

Finalmente, se concluye que es muy gratificante el trabajo que realizan las mujeres en las FFAA, enfrentándose a un mundo de hombres y una sociedad machista y patriarcal; estas mujeres entregan todo de sí mismas para demostrarse a sí mismas y al mundo que son iguales a los hombres, capaces de hacer lo mismo y de tener la misma posición social, económica, e histórica que los hombres.

Sin duda existe descontento para algunos soldados ya que las mujeres pueden representar una competencia, y puede resultar extraño para algunos el saber que ellas participan activamente de la institución militar, de la misma forma que cualquier hombre. Si superficialmente analizamos el status social de las mujeres, hay prejuicios al ver a una mujer involucrada en altos cargos de una empresa, hay estereotipos sexistas cuando hay mujeres ejerciendo un cargo político, y probablemente los haya por mucho tiempo. A medida de que cambia la sociedad cambian sus instituciones, por lo tanto las FFAA son y serán siempre el espejo de la sociedad.

\section{Referencias}

Amnesty International (2009). Las mujeres violadas durante el conflicto de Bosnia y Herzegovina sigue esperando justicia. Recuperado desde http://www.amnesty.org/fr/node/13374

Boyer, M. (2001). Gender, Violence and International Crisis. The Journal Of Conflict and Resolution, 45, 503-518.

Caprioli, M., \& Boyer, A. (2001). Gender, Violence, and International Crisis. The Journal of Conflict Resolution, 45 (4), 503-518. 
Chacón, R. (2008). Mujer Militar: su Inclusion en las Fuerzas Armadas. Quito: Publiasesores.

El Comercio. (2014). Los Ministros de Correa que dirijieron la Cartera de Defensa. Recuperado desde http://www.elcomercio.com/galerias/ ministros-correa-dirigieron-ministerio-defensa.html

Goldstein, J. S. (2001). War and Gender: How Gender Shapes the War System and Vice. Cambridge: Cambridge University Press.

Hopkins, J. (1968). Theological Language and the Nature of Man in Jean-Paul Sartre's Philosophy. Harvard Theological Review, 27-31.

Ichino, A., \& and Moretti, E. (2009). Biological Gender Differences, Absenteeism, and the Earnings Gap. American Economy Journal, 183218.

Donadio, M.. (2012). Ecuador. Atlas Comparativo de la Defensa en America Latina y Caribe. Buenos Aires: RESDAL.

Tessler, M. (1999). Further Tests of the Women and Peace Hypothesis: Evidence from Cross-National Survey. International Studies Quarter$l y, 43,519-531$. 